\title{
Comparison of the predictive value of area under the curve versus maximum serum concentration of intravenous tobramycin in cystic fibrosis patients treated for an acute pulmonary exacerbation
}

\author{
Katherine Landmesser ${ }^{1}$, Elizabeth Autry ${ }^{2}$, Brian Gardner ${ }^{2}$, Katherine Bosko ${ }^{2}$, Aric \\ Schadler ${ }^{3}$, and Robert Kuhn ${ }^{3}$ \\ ${ }^{1}$ University of Kentucky \\ ${ }^{2}$ University of Kentucky Medical Center \\ ${ }^{3}$ University of Kentucky College of Pharmacy
}

January 5, 2021

\begin{abstract}
OBJECTIVES: The primary objective of this study was to compare the therapeutic predictive value of area under the curve (AUC24) versus maximum concentration (Cmax) in cystic fibrosis (CF) patients receiving intravenous (IV) tobramycin for a Pseudomonas aeruginosa (PsA) acute pulmonary exacerbation (APE). Acute kidney injury (AKI) incidence and the relationship between time undetectable and efficacy were also assessed. METHODS: A retrospective review was conducted in patients aged at least one month with a diagnosis of CF receiving IV tobramycin for treatment of a PsA APE and admitted to the University of Kentucky between August 2015 and August 2019. Patients were excluded if they had no growth of PsA on sputum culture or if two post-dose tobramycin levels were not obtained following a dose adjustment of [?]20\%. RESULTS: A total of 44 pediatric and 107 adult patient encounters met inclusion criteria. In patients with therapeutic success ( $\mathrm{n}=91), 75.8 \%$ had an AUC24 [?]80 and $80.3 \%$ had a Cmax [?] 8 times the highest PsA minimal inhibitory concentration (MIC). There was a significant correlation between AUC24 and Cmax $(\mathrm{r} 2=0.727 ; \mathrm{p}<0.001)$. AKI incidence was significantly higher in patients receiving IV tobramycin dosed multiple times daily versus at least every 24 hours ( $50 \%$ versus $28.7 \%$; $=0.047)$. CONCLUSIONS: The results of this study indicate that both AUC24 and Cmax serve as relatively accurate predictors of tobramycin efficacy. Additionally, given the significant increase in incidence of AKI, multi-daily dosing of IV tobramycin should be used only in select pediatric and adult patients with $\mathrm{CF}$.
\end{abstract}

Title: Comparison of the predictive value of area under the curve versus maximum serum concentration of intravenous tobramycin in cystic fibrosis patients treated for an acute pulmonary exacerbation

Katherine B. Landmesser ${ }^{1}$, PharmD; Elizabeth B. Autry ${ }^{1,2}$, PharmD; Brian M. Gardner ${ }^{1,2}$, PharmD; Katherine A. Bosko ${ }^{2}$; Aric Schadler ${ }^{2}$, PhD; Robert J. Kuhn, PharmD ${ }^{1,2}$

${ }^{1}$ Department of Pharmacy, Kentucky Children's Hospital, Lexington, Kentucky; ${ }^{2}$ Department of Pharmacy, The University of Kentucky College of Pharmacy, Lexington, KY

Acknowledgments: Alexa Baskin

Research institution: University of Kentucky HealthCare

Running head: Pharmacokinetic analysis of IV tobramycin in CF

Keywords: Antibiotic therapy, cystic fibrosis, pharmacodynamics, pharmacokinetics, tobramycin

Corresponding Author Information: 
Katherine Landmesser: UK HealthCare 800 Rose Street; (859) 323-1281; kbl224@uky.edu

Abstract

OBJECTIVES: The primary objective of this study was to compare the therapeutic predictive value of area under the curve $\left(\mathrm{AUC}_{24}\right)$ versus maximum concentration $\left(\mathrm{C}_{\max }\right)$ in cystic fibrosis $(\mathrm{CF})$ patients receiving intravenous (IV) tobramycin for a Pseudomonas aeruginosa (PsA) acute pulmonary exacerbation (APE). Acute kidney injury (AKI) incidence and the relationship between time undetectable and efficacy were also assessed.

METHODS: A retrospective review was conducted in patients aged at least one month with a diagnosis of CF receiving IV tobramycin for treatment of a PsA APE and admitted to the University of Kentucky between August 2015 and August 2019 . Patients were excluded if they had no growth of PsA on sputum culture or if two post-dose tobramycin levels were not obtained following a dose adjustment of [?] $20 \%$.

RESULTS: A total of 44 pediatric and 107 adult patient encounters met inclusion criteria. In patients with therapeutic success $(\mathrm{n}=91), 75.8 \%$ had an $\mathrm{AUC}_{24}$ [?]80 and $80.3 \%$ had a $\mathrm{C}_{\max }$ [?]8 times the highest PsA minimal inhibitory concentration (MIC). There was a significant correlation between $\mathrm{AUC}_{24}$ and $\mathrm{C}_{\max }\left(\mathrm{r}^{2}=\right.$ 0.727; $\mathrm{p}<0.001)$. AKI incidence was significantly higher in patients receiving IV tobramycin dosed multiple times daily versus at least every 24 hours ( $50 \%$ versus $28.7 \%$; $=0.047$ ).

CONCLUSIONS: The results of this study indicate that both $\mathrm{AUC}_{24}$ and $\mathrm{C}_{\text {max }}$ serve as relatively accurate predictors of tobramycin efficacy. Additionally, given the significant increase in incidence of AKI, multi-daily dosing of IV tobramycin should be used only in select pediatric and adult patients with CF.

\section{INTRODUCTION:}

Pseudomonas aeruginosa (PsA) is frequently implicated as a cause of pulmonary infection and subsequent irreversible decline in lung function in patients with cystic fibrosis (CF) ${ }^{1-3}$. Intravenous (IV) aminoglycoside therapy has been shown to be relatively safe and efficacious, when monitored appropriately, for the treatment of PsA acute pulmonary exacerbations (APEs) in CF patients ${ }^{3}$. As aminoglycosides are concentration-dependent agents, the efficacy of therapy is enhanced when peak concentrations are maximized. Dosing strategies that maximize the peak concentration and minimize the trough concentration may enhance bactericidal activity while also reducing the risk of nephrotoxicity and ototoxicity ${ }^{4,5}$. Historically, a max concentration $\left(\mathrm{C}_{\max }\right)$ [?]8-foldthe minimal inhibitory concentration (MIC) has been the pharmacokinetic-pharmacodynamic (PK-PD) target utilized to predict IV aminoglycoside efficacy ${ }^{4-6}$. However, recent studies have suggested the benefit of utilizing area under the curve $\left(\mathrm{AUC}_{24}\right)$ to monitor total drug exposure to IV aminoglycoside therapy in CF patients, as both drug concentration and clearance are reflected ${ }^{7-8}$. This paradigm shift is supported by the fact that for our newest aminoglycoside, plazomicin, the FDA package labeling recommends monitoring the $\mathrm{AUC}_{24}$ : $\mathrm{MIC}$ ratio as it has been shown to best correlate with efficacy in animal and in vitro models against gram-negative pathogens ${ }^{9}$. Given the importance of balancing efficacy and toxicity, further studies assessing the clinical utility of $\mathrm{AUC}_{24}$ in $\mathrm{CF}$ patients receiving high-dose extended interval dosing of IV aminoglycoside therapy are warranted.

The primary objective of this study is to compare the predictive value of $\mathrm{AUC}_{24}$ versus $\mathrm{C}_{\max }$, in relation to mean change in forced expiratory volume over one second $\left(\mathrm{FEV}_{1}\right)$ in $\mathrm{CF}$ patients being treated for a PsA APE. Secondary objectives include: (1) to describe the incidence of acute kidney injury (AKI) and (2) to describe the relationship between time undetectable and the incidence of acute kidney injury (AKI) as well as the return of $\mathrm{FEV}_{1}$ to baseline.

\section{METHODS:}

\section{Eligibility criteria}

This was a single center retrospective review approved by the University of Kentucky Institutional Review Board (IRB). All patients aged one month or older with a diagnosis of CF who received IV tobramycin for treatment of a PsA APE during an admission to the University of Kentucky Healthcare System between 
August 1, 2015 and August 31, 2019 were evaluated for inclusion. Patients were excluded if they had no growth of PsA on sputum culture(s) prior to or during admission, if they had chronic kidney disease (CKD) prior to receiving the first dose of IV tobramycin, or if two post-dose drug levels were not obtained during admission to calculate patient-specific pharmacokinetic parameters. Further, patients were excluded from primary outcome analysis if two drug levels were not obtained following a dose adjustment of greater than $20 \%$ or following adjustment of the dosing interval secondary to fluctuating renal function.

Pharmacokinetic parameters were collected following any dose adjustment of IV tobramycin, and only the parameters collected from the final dose (i.e. based on clinical judgement, did not warrant further dose adjustment) were analyzed in the primary outcome analysis. Within the adult cohort, if a patient had $>4$ encounters over the four-year study period, only the first four encounters were collected and subsequently analyzed.

\section{Study design and procedures}

Information collected following review of the electronic medical record (EMR) included: baseline demographics, microbiologic data, lung function data, renal function data, and tobramycin dosing data. $\mathrm{AUC}_{24}, \mathrm{C}_{\max }$, time undetectable, and all additional patient-specific pharmacokinetic parameters were calculated from two post-dose tobramycin serum concentrations using Excel ${ }^{\circledR}$-based log-linear regression. Monitoring of tobramycin within this study typically adhered to our institution guidelines, which recommend obtaining levels four and ten hours following doses administered every 24 hours, and two and eight hours following doses administered every 12 hours. Per institution guidelines, patients typically had levels obtained after the third day of IV tobramycin therapy to allow for adequate rehydration. Doses were adjusted to achieve a target peak concentration of [?]12 mg/L (or eight to ten-fold the highest PsA MIC) and a trough concentration $<0.5 \mathrm{mg} / \mathrm{L}$. Institutional guidelines also recommend increasing the dosing frequency of IV tobramycin in patients who have a calculated time undetectable (concentration [?]0.5 mg/L) for longer than 8 hours within a 24-hour dosing interval.

\section{Definitions}

For the purposes of data analysis, a therapeutic $\mathrm{AUC}_{24}$ was defined as $80-120 \mathrm{mg} * \mathrm{hr} / \mathrm{L}$, which is consistent with previously cited targets for therapeutic drug monitoring of IV tobramycin in pediatric and adult CF patients ${ }^{7,10-11}$. $\mathrm{C}_{\max }$ was considered to be therapeutic if the concentration was equal to or exceeded eightfold the highest documented PsA MIC. All sputum cultures growing PsA obtained prior to and during admission were collected and analyzed to determine the highest MIC.

The surrogate marker of clinical efficacy utilized in this study was the categorization of end-of-treatment $\mathrm{FEV}_{1}$, from baseline. $\mathrm{FEV}_{1}$ was obtained on admission, immediately prior to discharge, and at the clinic appointment immediately following discharge. Due to variance in methods utilized to calculate the percent predicted $\mathrm{FEV}_{1}\left({ }_{\mathrm{pp}} \mathrm{FEV}_{1}\right.$ ) between outpatient and inpatient settings, $\mathrm{FEV}_{1}$ in liters was used to compare the

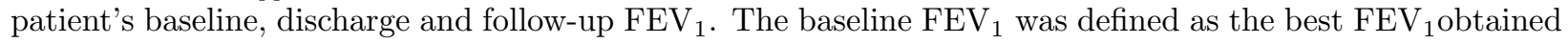
within the 6 months preceding admission. If the patient did not have pulmonary function tests (PFTs) obtained within 6 months of admission, the PFTs obtained at their previous annual clinic appointment were utilized to define baseline $\mathrm{FEV}_{1}$. A patient was considered to have returned to baseline if the $\mathrm{FEV}_{1}$ obtained at discharge was within $10 \%$ of baseline $\mathrm{FEV}_{1}$. Of note, if a patient was permitted to complete IV tobramycin therapy as outpatient, the $\mathrm{FEV}_{1}$ obtained at their follow-up appointment immediately following cessation of therapy was utilized for comparison to baseline.

The baseline serum creatinine was defined as the patient's lowest serum creatinine obtained within six months preceding admission. If the patient did not have a serum creatinine obtained within the six months preceding admission, the serum creatinine obtained at the patient's annual clinic appointment was considered baseline. The Kidney Disease Improving Global Outcomes (KDIGO) definition of an increase in serum creatinine by [?]0.3 mg/dL within 48 hours or an increase to [?]1.5 times baseline within 7 days was utilized to define patients with $\mathrm{AKI}^{12}$. 


\section{Statistical Analysis}

IBM SPSS Statistics version 25 was utilized for data analysis. Pearson's chi-square and Fisher's exact statistic were used to analyze nominal data as appropriate. Independent samples t-tests and independent samples differences of medians were utilized as appropriate for continuous data.

\section{RESULTS:}

\section{Patient characteristics}

A total of 44 pediatric encounters (19 patients) and 107 adult encounters (47 patients) met inclusion criteria, and were subsequently analyzed. Included patients were majority female $(56 \%)$ and Caucasian $(90.5 \%)$ with a median age of 24 years (range: $0.8-61$ years) (Table 1 ). One patient was excluded from analysis following development of AKI requiring discontinuation of IV tobramycin.

\section{Tobramycin dosing}

The mean daily dose of IV tobramycin was $12.3 \mathrm{mg} / \mathrm{kg}$ (standard deviation: 2.56 ) with the majority (84.8\%) of patients receiving therapy every 24 hours. Within the pediatric cohort, the mean daily dose of IV tobramycin was $18.9 \%$ higher $(13.8 \mathrm{mg} / \mathrm{kg}$ versus $11.6 \mathrm{mg} / \mathrm{kg})$ than the adult cohort, and more frequently dosed multiple times daily (43.2\% versus $3.7 \%$ ) (Table 2 ). This difference is likely reflective of the augmented renal function and faster drug clearance in pediatric patients, resulting in a longer time undetectable and subsequent dose adjustment to increase dosing frequency per institutional guidelines. In those that developed AKI, the median total daily dose of $13 \mathrm{mg} / \mathrm{kg}$ did not vary greatly from $12 \mathrm{mg} / \mathrm{kg}$ in those who did not develop AKI.

$A U C_{24}$ and $\mathrm{C}_{\max }$

Of the patients encounters with an $\mathrm{FEV}_{1}$ that returned to baseline or improved following IV tobramycin therapy $(\mathrm{n}=91), 75.8 \%$ had an $\mathrm{AUC}_{24}[?] 80 \mathrm{mg} * \mathrm{hr} / \mathrm{L}(\mathrm{p}=0.147)$ and $80.3 \%$ had a $\mathrm{C}_{\max }$ [?] 8 times the highest PsA MIC $(\mathrm{p}=0.065)$ (Table 3 ). The mean $\mathrm{C}_{\max }$ in patient encounters where the $\mathrm{FEV}_{1}$ returned to baseline was $24.2 \mathrm{mg} / \mathrm{L}$ versus $21.6 \mathrm{mg} / \mathrm{L}$ in those in which the $\mathrm{FEV}_{1}$ did not return to baseline $(\mathrm{p}=0.186)$. The mean $\mathrm{AUC}_{24}$ in patient encounters with an $\mathrm{FEV}_{1}$ that returned to baseline was $122.3 \mathrm{mg} / \mathrm{L}$ versus 108.7 $\mathrm{mg} / \mathrm{L}$ that did not return to baseline $(\mathrm{p}=0.141)$. Overall, there was a statistically significant correlation found between $\mathrm{AUC}_{24}$ and $\mathrm{C}_{\max }$ with an $\mathrm{r}^{2}$ value of $0.727(\mathrm{p}<0.001)($ Figure 1$)$.

\section{Time undetectable}

Within both cohorts, there was no statistical difference in time undetectable, defined as time for which tobramycin serum concentration was $<0.5 \mathrm{mg} / \mathrm{L}$, between patient encounters in which $\mathrm{FEV}_{1}$ returned to baseline, versus did not return to baseline, with median time undetectable of 9 and 8 hours, respectively $(\mathrm{p}=0.333)$. When comparing overall incidence of AKI, there was no difference in median time undetectable, with a median of 8 hours in encounters where the patient did and did not develop AKI.

\section{Safety}

Within both pediatric and adult cohorts, an increasing $\mathrm{AUC}_{24}$ or $\mathrm{C}_{\max }$ was not associated with subsequent development of AKI (Table 4 ). The mean $\mathrm{AUC}_{24}$ in encounters where patients developed AKI was 104.3 mg*hr/L versus a mean $\mathrm{AUC}_{24}$ of $119.6 \mathrm{mg} * \mathrm{hr} / \mathrm{L}$ in encounters in which the patient did not develop AKI $(\mathrm{p}=0.031)$. In patient encounters where the $\mathrm{AUC}_{24}$ was considered subtherapeutic $(<80 \mathrm{mg} * \mathrm{hr} / \mathrm{L})$, the rate of AKI development was $45.2 \%$ versus a rate of $28.3 \%$ in patient encounters where the $\mathrm{AUC}_{24}$ was considered therapeutic $\left(\mathrm{AUC}_{24}[?] 80 \mathrm{mg}^{*} \mathrm{hr} / \mathrm{L}\right)(\mathrm{p}=0.073)$. In patient encounters in which the $\mathrm{C}_{\max }$ was considered to be therapeutic $\left(\mathrm{C}_{\max }\right.$ [?] 8 to 10-fold the MIC), there was an associated incidence of AKI of $27 \%$ versus $36.4 \%$ in patient encounters with a $\mathrm{C}_{\max }$ considered subtherapeutic $(\mathrm{Cmax}<8$ to 10 -fold the MIC) $(\mathrm{p}=0.218)$. A statistically significant increase in incidence of AKI was noted in pediatric and adult patients receiving IV tobramycin dosed multiple times daily versus extended-interval dosing (at least every 24 hours) with $50 \%$ versus $28.7 \%$ of patient encounters developing AKI, respectively ( $\mathrm{p}=0.047)$ (Table 5 ). 


\section{DISCUSSION:}

\section{Efficacy}

The results of this study indicate that both $\mathrm{AUC}_{24}$ and $\mathrm{C}_{\max }$ serve as relatively accurate predictors of clinical efficacy, defined as a return to baseline or improvement in $\mathrm{FEV}_{1}$, with $75.8 \%$ and $80.3 \%$ of patients with therapeutic parameters achieving clinical efficacy, respectively. The lack of correlation between increasing $\mathrm{AUC}_{24}$ or $\mathrm{C}_{\max }$ and subsequent development of $\mathrm{AKI}$, in combination with the correlation of clinical efficacy and $\mathrm{AUC}_{24}[?] 80 \mathrm{mg}^{*} \mathrm{hr} / \mathrm{L}$, suggest benefit in targeting an $\mathrm{AUC}_{24}$ [?]80 mg*hr/L with minimal risk of additive toxicity.

Our institution guidelines recommend minimizing the calculated time undetectable, defined as time for which tobramycin serum concentration is $<0.5 \mathrm{mg} / \mathrm{L}$, to less than eight hours in an attempt to improve therapeutic efficacy. The lack of correlation between efficacy and time undetectable seen within this study may indicate that time undetectable does not play a significant role in influencing the efficacy of IV tobramycin in patients with CF being treated for a pulmonary exacerbation. Given the significantly increased incidence of AKI associated with multi-daily dosing of IV tobramycin found within this study, particularly in pediatric patients, multi-daily dosing to prevent the prolongation of time undetectable alone should be discouraged.

Safety

The utility of IV aminoglycoside therapy is often limited by the well-recognized risk of ototoxicity and nephrotoxicity ${ }^{3,10-11}$. Understanding the pharmacodynamics and pharmacokinetics of IV aminoglycoside therapy is essential to establish a balance between efficacy and toxicity. The results of this study unexpectedly demonstrated an increased incidence of AKI in the pediatric cohort, when compared to the adult cohort ( $45.5 \%$ versus $25.9 \%$ ), which was likely secondary to the increased incidence of multi-daily dosing of IV tobramycin in pediatric patients. Once-daily dosing of IV aminoglycoside therapy has previously been demonstrated to reduce nephrotoxicity, without sacrificing efficacy, in both pediatric and adult patients with CF ${ }^{13-19}$. Similar to previous studies, patients within our study who received IV tobramycin dosed multiple times daily had a significantly increased risk of AKI in comparison to patients receiving IV tobramycin dosed at least every 24 hours, with $50 \%$ versus $28.7 \%$ developing AKI, respectively $(\mathrm{p}=0.047)$. Our findings correlate with one Cochrane analysis comparing once versus multi-daily dosing of aminoglycoside therapy in pediatric and adult patients with $\mathrm{CF}$, which found a significantly lower rise in creatinine with oncedaily aminoglycoside therapy in children, perpetuating the recommendation to utilize once daily dosing of aminoglycosides, whenever possible, in pediatric patients ${ }^{16}$.

Given the lack of correlation between time undetectable and efficacy found within this study, modification of institution guidelines may be considered to discourage multi-daily dosing of IV tobramycin, thereby mitigating AKI incidence and improving clinical efficacy, in pediatric patients. Changes in dosing interval resulting in multi-daily dosing should not be recommended without considering both pharmacokinetic and clinical features of each patient. Practice changes, including the administration of once daily fluid boluses, have been incorporated within the children's hospital at our institution in the past year to mitigate the risk of AKI in pediatric CF patients receiving IV aminoglycoside therapy.

There were several limitations to this study including the retrospective nature which restricted data collection to information readily available within the electronic medical record (EMR). Additionally, prior to practice changes made within recent years, patients were often permitted to complete courses of IV therapy at home, limiting the ability to closely monitor IV tobramycin efficacy and toxicity as well as adherence to chest physiotherapy which may have impacted changes in $\mathrm{FEV}_{1}$. Lastly, alterations in pharmacokinetic parameters may have contributed to the lack of statistical significance found between therapeutic measurements of efficacy $\left(\mathrm{C}_{\max }\right.$ and $\left.\mathrm{AUC}_{24}\right)$ and changes in $\mathrm{FEV}_{1}$ found within this study. Of the 151 patient encounters analyzed, 56 patient encounters $(37 \%)$ had volumes of distribution (Vd) which were not considered physiologic, defined as a $\mathrm{Vd}$ between $0.3-0.65 \mathrm{~L} / \mathrm{kg}$. Non-physiologic volumes of distribution may result in falsely altered $\mathrm{C}_{\max }$ and $\mathrm{AUC}_{24}$ measurements, resulting in lack of correlation between these measured variables and clinical success, defined as return of $\mathrm{FEV}_{1}$ to baseline. Overall 71 patient encounters (49\%) 
had measured $\mathrm{AUC}_{24}$ [?]80 and $\mathrm{C}_{\max }$ [?]8 times the highest Pseudomonas MIC, resulting in the need for a larger patient population than what was originally anticipated to establish statistical significance.

\section{Conclusions}

The results of this study indicate that utilization of $\mathrm{AUC}_{24}$, in combination with $\mathrm{C}_{\max }$, may be beneficial when monitoring IV tobramycin in patients with CF admitted for an APE. The lack of correlation between increasing $\mathrm{AUC}_{24}$ or $\mathrm{C}_{\max }$ and subsequent development of $\mathrm{AKI}$, in combination with the correlation of clinical efficacy and $\mathrm{AUC}_{24}[?] 80 \mathrm{mg}$ hr/L, suggest benefit in targeting an $\mathrm{AUC}_{24}$ [?] $80 \mathrm{mg} * \mathrm{hr} / \mathrm{L}$ with minimal risk of additive toxicity. Overall, given the significant correlation between $\mathrm{AUC}_{24}$ and $\mathrm{C}_{\max }$ found within both cohorts, $\mathrm{AUC}_{24}$ may be utilized, in combination with $\mathrm{C}_{\max }$, to enhance efficacy of IV tobramycin therapy in $\mathrm{CF}$ patients being treated for an acute pulmonary exacerbation.

Additionally, given the significant increase in incidence of AKI with multi-daily dosing of tobramycin, in combination with the lack of correlation between time undetectable and efficacy or toxicity found within this study, utilization of once-daily dosing of IV tobramycin should be encouraged in both pediatric and adult patients with $\mathrm{CF}$.

\section{CITATIONS:}

1. Bhagirath A, Li Y, Somayajula D, Dadashi M, Badr S, Duan K. Cystic fibrosis lung environment and Pseudomonas aeruginosa infection. BMC pulmonary medicine . 5 Dec. 2016;16(1): 174.

2. Kerem E, Viviani L, Zolin A, MacNeill S, Hatziagorou E, Ellemunter H, Drevinek P, Gulmans V, Krivec U, Olesen H, ECFS Patient Registry Steering Group. Factors associated with FEV1 decline in cystic fibrosis: analysis of the ECFS Patient Registry. European Respiratory Journal . 2014 Jan;43(1):125-33.

3. Flume PA, Mogayzel PJ, Robinson KA, Goss CH, Rosenblatt RL, Kuhn RJ, Marshall BC, Clinical Practice Guidelines for Pulmonary Therapies Committee. Cystic Fibrosis Pulmonary Guidelines: Treatment of Pulmonary Exacerbations. Am J Respir Crit Care Med . 2009 Nov 1;180(9):802-8.

4. Hyatt JM, McKinnon PS, Zimmer GS, Schentag JJ. The importance of pharmacokinetic/pharmacodynamic surrogate markers to outcome. Focus on antibacterial agents. Clin Pharmacokinet . $1995 \mathrm{Feb} ; 28(2): 143-60$.

5. Kelvin H, Tan V, Mulheran M, Knox AJ, Smyth AR. Aminoglycoside Prescribing and Surveillance in Cystic Fibrosis. Am J Respir Crit Care Med. 2003;167: 819-823.

6. Bates RD, Nahata MC, Jones JW, Mccoy K, Young G, Cox S, Barson W. Pharmacokinetics and safety of tobramycin after once-daily administration in patients with cystic fibrosis. Chest . 1997;112 (5): $1208-1213$.

7. Coulthard KP, Peckham DG, Conway SP, Smith CA, Bell J, Turnidge J. Therapeutic drug monitoring of once daily tobramycin in cystic fibrosis - caution with trough concentrations. J Cyst Fibros . 2007 Apr;6(2):125-30.

8. Barclay ML, Kirkpatrick CM, Begg EJ. Once daily aminoglycoside therapy. Is it less toxic than multiple daily doses and how should it be monitored? Clin Pharmacokinet . 1999 Feb;36(2):89-98.

9. ZEMDRI(r) (plazomicin) injection package insert. Achaogen Inc. Revised 1/2020.

10. Rybak MJ, Abate BJ, Kang SL, Ruffing MJ, Lerner SA, Drusano GL. Prospective evaluation of the effect of an aminoglycoside dosing regimen on rates of observed nephrotoxicity and ototoxicity. Antimicrob Agents Chemother. 1999 Jul;43(7):1549-55.

11. Prescott WA, Nagel JL. Extended-Interval Once-Daily Dosing of Aminoglycosides in Adult and Pediatric Patients with Cystic Fibrosis.Pharmacotherapy 2010;30(1):95-108.

12. Khwaja A: Kidney Disease Improving Global Outcomes (KDIGO) Clinical Practice Guideline for Acute Kidney Injury. Nephron Clin Pract.2012;120:179-184.

13. VandenBussche HL, Homnick DN. Evaluation of Serum Concentrations Achieved with an Empiric Once-Daily Tobramycin Dosage Regimen in Children and Adults With Cystic Fibrosis. J Pediatr Pharmacol Ther. 2012 Jan;17(1):67-77.

14. Young DC, Zobell JT, Stockmann C, Waters CD, Ampofo K, Sherwin C, Spigarelli MG. Optimization of Anti-Pseudomonal Antibiotics for Cystic Fibrosis Pulmonary Exacerbations: V. Aminoglycosides. 
Pediatric Pulmonology. 2013 Nov;48(11):1047-61.

15. Zobell JT, Epps K, Kittell F, Sema C, McDade EJ, Peters SJ, Duval MA, Pettit RS. Tobramycin and Beta-Lactam Antibiotic Use in Cystic Fibrosis Exacerbations: A Pharmacist Approach. J Pediatr Pharmacol Ther. 2016;21(3):239-246.

16. Smyth AR, Bhatt J, Nevitt SJ. Once-daily versus multiple-daily dosing with intravenous aminoglycosides for cystic fibrosis. Cochrane Database of Systematic Reviews 2017 (3).

17. Master V, Roberts GW, Coulthard KP, Baghurst PA, Martin A, Roberts ME, Onishko CR, Martin AJ, Linke RJ, Holmes M, Jarvinen A, Kennedy D, Colebatch KA, Hansman D, Parsons DW. Efficacy of once-daily tobramycin monotherapy in acute pulmonary exacerbation of cystic fibrosis. Pediatr Pulmonol. 2001 May;31(5):367-76.

18. Whitehead A, Conway SP, Etherington C, Caldwell NA, Setchfield N, Bogle S. Once-daily tobramycin in the treatment of adult patients with cystic fibrosis. Eur Respir J. 2002;19:303-309.

19. Smyth A, Tan KH, Hyman-Taylor P, Mulheran M, Lewis S, Stableforth D, Prof Knox A; TOPIC Study Group. Once versus three-times daily regimens of tobramycin treatment for pulmonary exacerbations of cystic fibrosis-the TOPIC study: a randomised controlled trial.Lancet . 2005 Feb 12-18;365(9459):5738.

Table 1: Patient Demographics

\begin{tabular}{ll}
\hline Patient Demographics (n=152) & Patient Demographics (n=152) \\
Female (\%) & $85(56 \%)$ \\
Median Age, years [Range] & $24[0.8-61]$ \\
\% Hispanic & $4.5 \%$ \\
\% Caucasian & $90.5 \%$ \\
\% Non-Hispanic African American & $4.5 \%$ \\
\hline
\end{tabular}

Table 2: Tobramycin Dosing

\begin{tabular}{ll}
\hline Adult and Pediatric Cohorts $(\mathbf{n}=\mathbf{1 5 1})$ & Adult and Pediatric Cohorts $(\mathbf{n}=\mathbf{1 5 1})$ \\
Mean Daily Dose, mg/kg (Standard Deviation) & $12.3(2.56)$ \\
Dosed q36h (\%) & $1(0.7)$ \\
Dosed q24h (\%) & $128(84.8)$ \\
Dosed q18h (\%) & $5(3.3)$ \\
Dosed q12h (\%) & $17(11.3)$ \\
Adult Cohort $(\mathbf{n = 1 0 7 )}$ & Adult Cohort $(\mathbf{n = 1 0 7 )}$ \\
Mean Daily Dose, mg/kg (Standard Deviation) & $11.6(2.36)$ \\
Dosed 36h (\%) & $1(0.9)$ \\
Dosed q24h (\%) & $102(95.4)$ \\
Dosed q18h (\%) & $3(2.8)$ \\
Dosed q12h (\%) & $1(0.9)$ \\
Pediatric Cohort $(\mathbf{n}=\mathbf{4 4})$ & Pediatric Cohort $(\mathbf{n}=\mathbf{4 4 )}$ \\
Mean Daily Dose, mg/kg (Standard Deviation) & $13.8(2.43)$ \\
Dosed q24h (\%) & $25(56.8)$ \\
Dosed q18h (\%) & $2(4.6)$ \\
Dosed q12h (\%) & $17(38.6)$ \\
\hline
\end{tabular}

Table 3: $\mathrm{AUC}_{24}$ and $\mathrm{C}_{\max }$ Correlation with $\mathrm{FEV}_{1}$

$\mathrm{AUC}_{24}<80(\mathrm{n}=26)$

Returned to Baseline, n (\%)
$\mathrm{AUC}_{24}<80(\mathrm{n}=26)$

$16(61.5)$ 
Did NOT Return to Baseline, n (\%)

$\mathrm{AUC}_{24}$ [?] $80(\mathrm{n}=99)$

Returned to Baseline, n (\%)

Did NOT Return to Baseline, n (\%)

Pearson Chi-Square 2-sided significance $\left(\mathrm{AUC}_{24}\right): \mathrm{p}=0.147$

$\mathrm{C}_{\max }<8$-fold the highest PsA MIC $(\mathrm{n}=64)$

Returned to Baseline, n (\%)

Did NOT Return to Baseline, n (\%)

$\mathrm{C}_{\max }$ [?] 8-fold the highest PsA MIC $(\mathrm{n}=61)$

Returned to Baseline, n (\%)

Did NOT Return to Baseline, n (\%)

Pearson Chi-Square 2-sided significance $\left(\mathrm{C}_{\max }\right): \mathrm{p}=0.065$
$10(38.5)$

$\mathrm{AUC}_{24}$ [?] $80(\mathrm{n}=99)$

$75(75.8)$

$24(24.2)$

Pearson Chi-Square 2-sided significance $\left(\mathrm{AUC}_{24}\right): \mathrm{p}=0.147$

$\mathrm{C}_{\max }<8$-fold the highest PsA MIC $(\mathrm{n}=64)$

$42(65.6)$

$22(34.4)$

$\mathrm{C}_{\max }$ [?] 8-fold the highest PsA MIC $(\mathrm{n}=61)$

$49(80.3)$

$12(19.7)$

Pearson Chi-Square 2-sided significance $\left(\mathrm{C}_{\max }\right): \mathrm{p}=0.065$

Table 4: $\mathrm{AUC}_{24}$ and $\mathrm{C}_{\max }$ Correlation with AKI

\begin{tabular}{|c|c|}
\hline $\mathrm{AUC}_{24}<80(\mathrm{n}=31)$ & $\mathrm{AUC}_{24}<80(\mathrm{n}=31)$ \\
\hline Developed AKI, n (\%) & $14(45.2)$ \\
\hline Did NOT Develop AKI, n (\%) & $17(54.8)$ \\
\hline $\mathrm{AUC}_{24}[?] 80(\mathrm{n}=120)$ & $\mathrm{AUC}_{24}[?] 80(\mathrm{n}=120)$ \\
\hline Developed AKI, n (\%) & $34(28.3)$ \\
\hline Did NOT Develop AKI, n (\%) & $86(71.7)$ \\
\hline Pearson Chi-Square 2-sided significance: $\mathrm{p}=0.147$ & Pearson Chi-Square 2-sided significance: $\mathrm{p}=0.147$ \\
\hline $\mathrm{C}_{\max }<8$-fold the highest PsA MIC $(\mathrm{n}=77)$ & $\mathrm{C}_{\max }<8$-fold the highest PsA MIC $(\mathrm{n}=77)$ \\
\hline Developed AKI, n (\%) & $28(36.4)$ \\
\hline Did NOT Develop AKI, n (\%) & $49(63.6)$ \\
\hline $\mathrm{C}_{\max }[?]$ 8-fold the highest PsA MIC $(\mathrm{n}=74)$ & $\mathrm{C}_{\max }$ [?] 8-fold the highest PsA MIC $(\mathrm{n}=74)$ \\
\hline Developed AKI, n (\%) & $20(27)$ \\
\hline Did NOT Develop AKI, n (\%) & $54(73)$ \\
\hline Pearson Chi-Square 2-sided significance: $\mathrm{p}=0.218$ & Pearson Chi-Square 2-sided significance: $\mathrm{p}=0.218$ \\
\hline
\end{tabular}

Table 5: Correlation between Dosing and AKI Incidence

\begin{tabular}{ll}
\hline Median Total Daily Dose $(\mathrm{n}=151)$ & Median Total Daily Dose $(\mathrm{n}=151)$ \\
Developed AKI, mg/kg (standard deviation) & $13(3.02)$ \\
Did NOT Develop AKI, mg/kg (standard deviation) & $12(2.29)$ \\
Dosing Frequency [?] Every 24 Hours (n=129) & Dosing Frequency [?] Every 24 Hours (n=129) \\
Developed AKI, n (\%) & $37(28.7 \%)$ \\
Did NOT Develop AKI, n (\%) & $92(71.3 \%)$ \\
Dosing Frequency < Every 24 Hours $(\mathrm{n}=22)$ & Dosing Frequency < Every 24 Hours $(\mathrm{n}=22)$ \\
Developed AKI, n (\%) & $11(50 \%)$ \\
Did NOT Develop AKI, n (\%) & $11(50 \%)$ \\
Pearson Chi-Square 2-sided significance: $\mathrm{p}=0.047$ & Pearson Chi-Square 2-sided significance: $\mathrm{p}=0.047$ \\
\hline
\end{tabular}




\begin{tabular}{|c|c|c|c|}
\hline \multicolumn{4}{|c|}{ Correlations } \\
\hline & & Final AUC & Cmax \\
\hline \multirow[t]{3}{*}{ Final AUC } & Pearson Correlation & 1 & $.727^{\circ}$ \\
\hline & Sig. (2-tailed) & & .000 \\
\hline & $\mathrm{N}$ & 151 & 151 \\
\hline \multirow[t]{3}{*}{ Cmax } & Pearson Correlation & $.727^{*}$ & 1 \\
\hline & Sig. (2-tailed) & .000 & \\
\hline & $\mathrm{N}$ & 151 & 151 \\
\hline
\end{tabular}

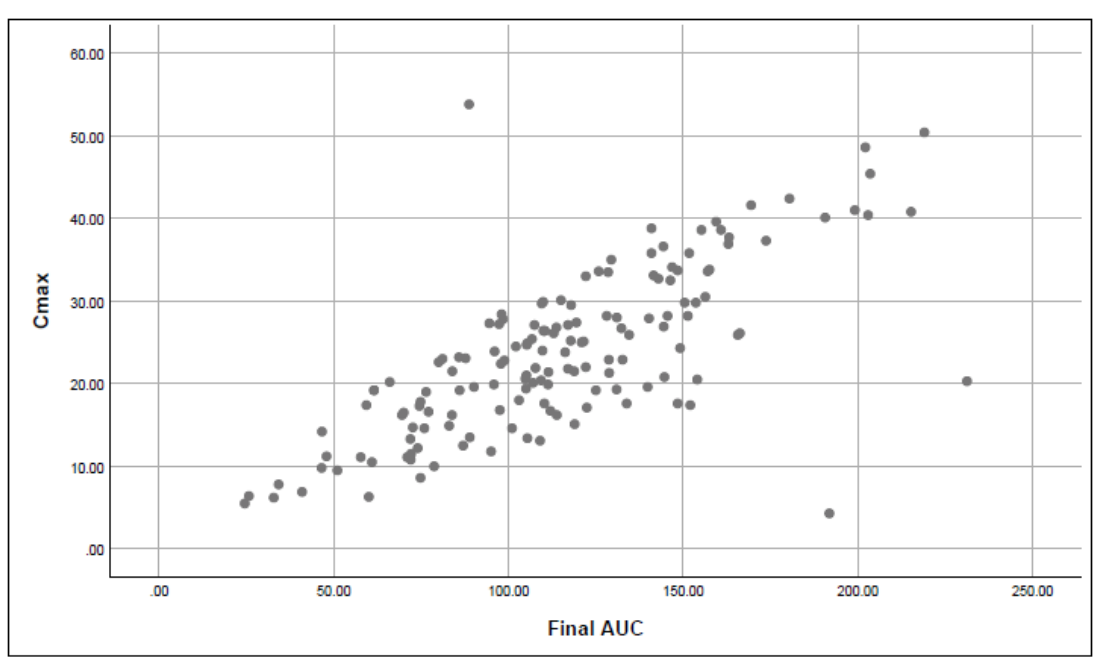

Figure 1: $\mathrm{AUC}_{24}$ and $\mathrm{C}_{\max }$ Correlation 